\title{
Successful Cross-circulation Stent-Retriever Embolectomy Through Posterior Communicating Artery for Acute MCA Occlusion by Using Trevo XP ProVue
}

\author{
Seul Kee Kim, MD², Byung Hyun Baek, MD', Tae Wook Heo, MD', Woong Yoon, MD'
}

Acute ischemic stroke due to embolic occlusion of the middle cerebral artery (MCA) in patients with chronic ipsilateral internal carotid artery (ICA) occlusion is quite rare. Several previous reports demonstrated that intra-arterial (IA) thrombolytic therapy or aspiration thrombectomy using the cross-circulation technique via an alternative collateral pathway is feasible in acute stroke patients with an unfavorable direct route to the occluded sites. However, stent-retriever embolectomy via the cross-circulation approach has not been reported in the literature. The present paper reports the first case of successful stent-retriever embolectomy for acute MCA occlusion via the patent posterior communicating artery (PComA) by using Trevo XP ProVue stent-retriever in a patient with acute MCA stroke and chronic occlusion at the origin site of the ipsilateral ICA.

Key Words: Cross-circulation; Acute ischemic stroke; Stent-retriever embolectomy

Several recent randomized controlled trials have shown that stent-retriever embolectomy in addition to standard care was associated with improvement of functional outcome in patients with acute anterior

'Department of Radiology, Chonnam National University Hospital, Gwangju, Korea

${ }^{2}$ Department of Radiology, Chonnam National University Hwasun Hospital, Jeollanam-do, Korea

Received February 12, 2016;

accepted after revision February 13, 2016.

Correspondence to: Woong Yoon, MD, Department of Radiology, Chonnam National University Hospital, 42 Jebong-ro, Dong-gu, Gwangju 61469, Korea.

Tel. 82.62.220.5746 Fax. 82.62.226.4380

E-mail: radyoon@jnu.ac.kr

This is an Open Access article distributed under the terms of the Creative Commons Attribution Non-Commercial License (http://creativecommons.org/licenses/by-nc/3.0) which permits unrestricted non-commercial use, distribution, and reproduction in any medium, provided the original work is properly cited. circulation stroke [1]. Acute ischemic stroke due to embolic occlusion of middle cerebral artery (MCA) in patients with chronic ipsilateral internal carotid artery (ICA) occlusion is quite rare, and there is currently no standardized treatment approach for these patients. Several previous reports demonstrated that intra-arterial (IA) pharmacologic thrombolysis or aspiration thrombectomy using the cross-circulation technique for treatment of acute ischemic stroke via circle of Willis arteries is feasible in patients with unfavorable direct route to the occluded sites. We present the first case report of the acute MCA occlusion in a patient with acute stroke who had a chronically occluded ipsilateral ICA, which was treated by stent-retriever embolectomy via the patent posterior communicating artery (PComA). Excellent angiographic and clinical outcomes were obtained without any complication in our patient. 


\section{Seul Kee Kim, et al.}

\section{CASE REPORT}

A 57-year-old right-handed man with a history of hypertension and current smoking presented 60 minutes after acute onset of left hemiparesis and dysarthria. His National Institute of Health Stroke Scale (NIHSS) Score was 15 at admission. Unenhanced computed tomography (CT) of his brain demonstrated a dense right MCA sign without intracranial hemorrhage. He was treated with $67 \mathrm{mg}$ of intravenous recombinant tissue plasminogen activator (rtPA). Emergent magnetic resonance imaging (MRI) of the brain was performed using a 1.5T unit (Signa HDxt; GE Medical Systems, Milwaukee, WI). Diffusion-weighted imaging showed an area of restricted diffusion in the right basal ganglia (Fig. 1A). The brain MRA revealed a complete occlusion at the mid M1 segment of the right MCA (Fig. 1B). Anterior cerebral circulation was supplied from the posterior circulation via the right PComA and left persistent trigeminal artery. There were no flow-related enhancements of the bilateral ICAs. Despite intravenous infusion of rtPA for one hour, no neurologic improvement was observed. Therefore, subsequent endovascular therapy was performed with conscious sedation 140 minutes after symptom onset.

A right common carotid artery (CCA) angiogram demonstrated a chronic occlusion at the origin site of the right ICA with a small stump (Fig. 1C). A left CCA angiogram also showed a chronic occlusion at the origin site of the left ICA (not shown). The right vertebral artery (VA) angiogram confirmed an occlusion at the mid M1 segment of the right MCA, which was reconstituted by a patent right PComA (Fig. 1D). The left side anterior circulation was supplied by a left persistent trigeminal artery. We decided to perform a stent-retriever embolectomy for the MCA occlusion through the patent PComA. An 8-F guide catheter was placed in the right VA. Using a standard technique, a microcatheter with a 0.021 -inch diameter was navigated distal to the clot over a 0.014 -inch microwire to the right MCA through the right PComA. A $4 \times 20$ $\mathrm{mm}$ Trevo XP ProVue stent (Stryker, Kalamazoo, MI, USA) was then introduced through the microcatheter and fully deployed across the occluded right MCA (Fig. $1 \mathrm{E}$ and $1 \mathrm{~F})$. After the stent was maintained in place for one minute, the fully deployed stent and delivery microcatheter were slowly pulled back together and withdrawn outside the body through the guide catheter. A large red thrombus was retrieved with a Trevo stent on a single pass (Fig. 1G). During clot retrieval, contin- uous manual aspiration with a $50-\mathrm{mL}$ syringe at the guide catheter was performed. A post-thrombectomy control angiogram demonstrated complete revascularization of the occluded MCA (Fig. 1H). Time to revascularization, defined as the time from the femoral access to achievement of revascularization, was 18 minutes.

The patient had significant improvement in neurological function immediately after the embolectomy. CT of the brain performed 2 days later demonstrated acute infarction in the right basal ganglia without intracranial hemorrhage. At the time of discharge after 10 days after stroke, the patient's NIHSS score was 2 .

\section{DISCUSSION}

Advances in endovascular technology permit novel approaches for recanalization when direct access to the occluded sites is not possible. This case demonstrates the feasibility and safety of cross-circulation stent retriever embolectomy via a patent $\mathrm{PComA}$ to reach a MCA thrombus in the setting of a chronic proximal ICA occlusion.

Access to an intracranial lesion crossing from the proximal major cerebral artery through patent communicating arteries, which is called a trans-circulation technique or cross-circulation technique, was first introduced for aneurysmal coiling [2, 3]. Improved design of microwires, microcatheters, stent systems, and guide catheters has facilitated the use of these techniques for endovascular treatment in patients with acute ischemic stroke when direct access to the lesions was impossible. Hui et al. first reported this crosscirculation technique for the treatment of acute ischemic stroke in a patient with a MCA occlusion via the posterior circulation approach using a patent PComA with aspiration thrombectomy [4]. Later on, Liu et al. reported successful anterior-posterior revascularization of a basilar artery occlusion through a patent PComA with IA thrombolysis and aspiration thrombectomy [5]. Ozdemir et al. has demonstrated that MCA occlusions can be recanalized with IA thrombolysis via a patent PComA, but they achieved complete recanalization in only $25 \%$ of eight patients [6]. In the present case report, we present the first case using the crosscirculation technique to perform stent-retriever embolectomy for treatment of the acute MCA occlusion in association with chronically occluded cervical ICA via the patent PComA in a patient with acute ischemic stroke. Direct access to the occluded MCA through the chronically occluded ICA might be 


\section{Cross-circulation Stent-Retriever Embolectomy for Acute MCA Occlusion}
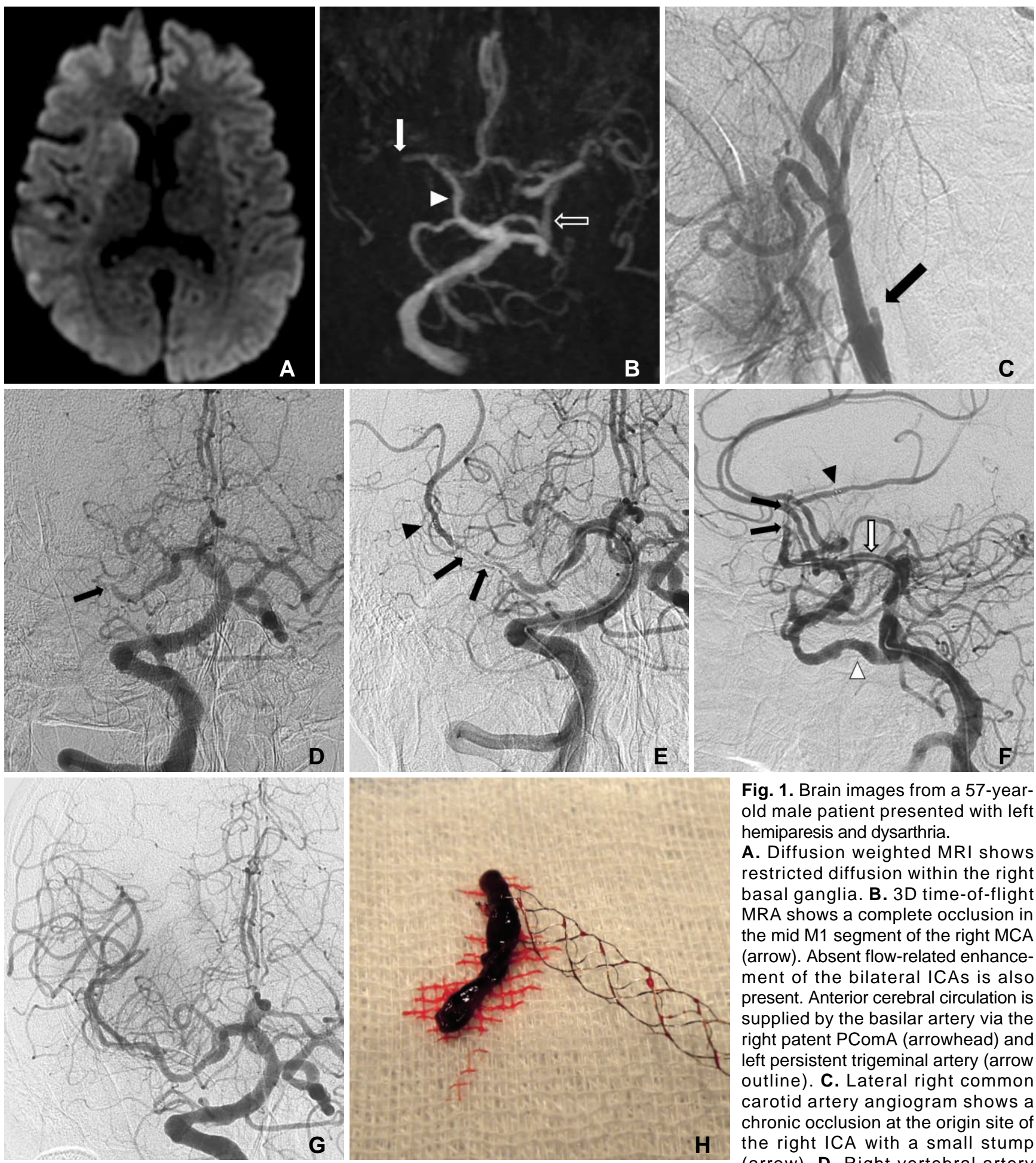

Fig. 1. Brain images from a 57-yearold male patient presented with left hemiparesis and dysarthria.

A. Diffusion weighted MRI shows restricted diffusion within the right basal ganglia. B. 3D time-of-flight MRA shows a complete occlusion in the mid M1 segment of the right MCA (arrow). Absent flow-related enhancement of the bilateral ICAs is also present. Anterior cerebral circulation is supplied by the basilar artery via the right patent PComA (arrowhead) and left persistent trigeminal artery (arrow outline). C. Lateral right common carotid artery angiogram shows a chronic occlusion at the origin site of the right ICA with a small stump (arrow). D. Right vertebral artery angiogram demonstrating an occlusion in the mid M1 segment of the right MCA (arrow). E and F. A $4 \times 20 \mathrm{~mm}$ Trevo XP ProVue retriever stent is deployed over the whole length of the thrombus in the right MCA through the right PComA (white arrow). Stent has three radiopaque distal markers (black arrowheads). Anterior-posterior and lateral vertebral artery angiogram following stent deployment, shows immediate distal flow of the right MCA. Note the filling defect in the M1 segment representing a trapped thrombus within the stent struts (black arrows). White arrowhead indicates the left persistent trigeminal artery. G. Angiogram after stent retrieval embolectomy shows complete revascularization in the right MCA territory. $\mathbf{H}$. Photograph demonstrates a large red clot retrieved with a Trevo stent. 


\section{Seul Kee Kim, et al.}

considered risky and time-consuming. Early recanalization of an occluded artery plays a critical role in the prognosis of a patient with acute ischemic stroke. Although we did not encounter any technical difficulty or complication when performing embolectomy across the PComA, the concern regarding advancement of endovascular devices through communicating arteries with a small diameter, such as dissection, rupture or embolic stroke, should be addressed. This risk may be increased in patients with advanced atherosclerotic changes in the proximal carotid and vertebral arteries [6].

We performed stent-retriever embolectomy using the Trevo XP ProVue stent. The Trevo XP ProVue is constructed of a single closed-cell nitinol hypotube and has no free margins, which might make retrieval of the device atraumatic [7]. These features of the stent might have contributed to the success of revascularization without vascular injury in our case.

In conclusion, our case demonstrates the feasibility and safety of stent-based embolectomy by using the cross-circulation technique via a Wills circle artery. Knowledge of an alternative vascular approach and the characteristics of the endovascular device is essential to achieve successful revascularization in acute stroke patients with an intracranial large vessel occlusion without a direct access route to the occlusion site.

\section{References}

1. Song D, Cho AH. Previous and recent evidence of endovascular therapy in acute ischemic stroke. Neurointervention 2015;10:5159

2. Moret J, Ross IB, Weill A, Piotin M. The retrograde approach: a consideration for the endovascular treatment of aneurysms. AJNR Am J Neuroradiol 2000;21:262-268

3. Albuquerque FC, Gonzalez LF, Hu YC, Newman CB, McDougall CG. Transcirculation endovascular treatment of complex cerebral aneurysms: technical considerations and preliminary results. Neurosurgery 2011;68:820-829; discussion 829-830

4. Hui FK, Narayanan S, Cawley CM. Posterior-to-anterior circulation access using the Penumbra Stroke System for mechanical thrombectomy of a right middle cerebral artery thrombus. World Neurosurg 2010;73:17-21

5. Liu W, Kung DK, Mahaney KB, Rossen JD, Jabbour PM, Hasan DM. Anterior-to-posterior circulation approach for mechanical thrombectomy of an acutely occluded basilar artery using the penumbra aspiration system. World Neurosurg 2012;77:398 E317320

6. Ozdemir O, Bussière M, Leung A, Gulka I, Lee D, Chan R, et al. Intra-arterial thrombolysis of occluded middle cerebral artery by use of collateral pathways in patients with tandem cervical carotid artery/middle cerebral artery occlusion. AJNR Am J Neuroradiol 2008;29:1596-1600

7. Leishangthem L, Satti SR. Vessel perforation during withdrawal of Trevo ProVue Stent retriever during mechanical thrombectomy for acute ischemic stroke. J Neurosurg 2014;121:995-998 